\title{
Primary defects of the hand with intrinsic paralysis
}

\author{
J W BRANDSMA \& J G ANDERSEN \\ All Africa Leprosy and Rehabilitation Training Centre (ALERT), \\ PO Box 165, Addis Ababa, Ethiopia
}

\section{Accepted for publication 16 January 1984}

\begin{abstract}
Summary The primary defects of the hand that has suffered intrinsic paralysis are described.

The extent to which these defects are corrected by standard tendon transfer operations is discussed.
\end{abstract}

\section{Introduction}

The intrinsic muscles of the hand are all innervated by either the ulnar or the median nerve. Although there are some variations in the pattern of innervation, the commonly found pattern is for the ulnar nerve to innervate the hypothenar muscles, the interossei, the two ulnar lumbrical muscles, adductor pollicis and the ulnar head of flexor pollicis brevis. The median nerve innervates the abductor pollicis brevis, radial head of flexor pollicis brevis, opponens pollicis and the two radial lumbrical muscles.

In the leprosy patient both isolated ulnar paralysis, with or without paralysis of flexor carpi ulnaris and the ulnar half of flexor digitorum profundus, and combined ulnar and 'low' median paralysis are commonly found. Distinctly less common are median paralysis, either isolated or in combination with ulnar paralysis as a 'high' median paralysis with paralysis of the extrinsic flexor muscles.

Radial paralysis is less common, but when seen is usually found in combination with intrinsic paralysis in a variety of patterns. This naturally plays an important role in the planning of surgical restoration, but this is outside the scope of this paper.

The ulnar nerve may become damaged by leprosy either proximal to the ulnar epicondylar canal or just proximal to Guyon's canal. The median nerve is usually damaged just proximal to the carpal tunnel.

Paralysis of the two ulnar innervated extrinsic muscles plays little or no role in the development of the primary defects, the immediate effects of paralysis, but may influence the development of secondary defects.

The functions of the hand may be summarized as follows:

1 Prehensile movements. Napier ${ }^{1}$ defined two basic prehensile movements, power grip and precision grip. Landsmeer ${ }^{2}$ preferred to call the latter precision handling. 
Table 1. Primary defects of ulnar and median palsy

Defect

\begin{tabular}{|c|c|}
\hline & nar \\
\hline & Incomplete finger extension (clawing) \\
\hline & 'Reversed' pattern of finger closing \\
\hline & Loss of antepulsion, 4th and 5th metacarpa \\
\hline & Loss of abduction and adduction of fingers \\
\hline & Decreased grip-and-pinch strength \\
\hline & Loss of stability of the thumb \\
\hline
\end{tabular}

Responsible muscles

Median

7 Loss of opposition

Interossei-lumbricals

Interossei-lumbricals

Hypothenar muscles

Interossei-hypothenar

Interossei-hypothenar-adductor pollicis

Flexor pollicis-adductor pollicis

2 Non-prehensile movements, pushing, lifting and tapping movements, e.g. those employed when typing or playing the piano.

3 The hand is the chief organ of touch. ${ }^{3}$ As such it also monitors the muscle power by a sensory feedback. ${ }^{4}$ As an example of this it may be mentioned that many patients with loss of sensation but intact motor function complain of a weak hand.

4 The hand is the chief organ of communication, next to the voice. ${ }^{3}$ We can mention the importance of the warning hand, the greeting hand, the signalling hand, e.g. used when hitchhiking and the manifold hand gestures that vividly express the personality of the person.

Confronted with a hand with intrinsic paralysis, a surgeon will readily recognize clawing of the fingers and thenar paralysis with loss of opposition of the thumb. He may not realize that other defects are present that influence the function of the hand.

Paralysis of the intrinsic muscles is responsible for seven primary defects (Table 1).

\section{Primary defects}

\section{Incomplete digital extension}

In pure ulnar paralysis the ring and small fingers inevitably develop a claw deformity. In most cases the index and middle fingers af ter a period of 'latent' clawing, where the 'lumbrical' position may be achieved, but where the fingers slip into clawing when power grip is attempted, will develop manifest clawing. The relative role of the interosseus and lumbrical muscles in the development of clawing has been discussed. ${ }^{5}$ Four-finger clawing was noticed in most hands with ulnar palsy only, indicating that the lumbricals alone could not prevent fingerclawing in the index and middle fingers. On the other hand clawing of index and middle finger was never encountered in pure median paralysis.

Incomplete finger extension is a obvious cosmetic defect. Functionally it prevents full opening of the hand with inability to close the hand round an object with equal pressure distribution over the whole volar surface of the fingers. The obvious claw position, metacarpophalangeal hyperextension and interphalangeal flexion makes greetings embarrassing, which may have serious social effects.

\section{Reversed pattern of closing}

It is stated ${ }^{6}$ that the patient with intrinsic paralysis will close his hand in reverse pattern, i.e. distal interphalangeal flexion, followed by proximal interphalangeal flexion and finally metacarpophalangeal flexion, exactly the opposite of the normal sequence of closing. In power grip the major part 
of all the pressure will fall on the tips of the fingers. Add to that the usually found loss of sensory feed back and we have the logical explanation of the progressive absorption of the fingers in the anaesthetic hand.

\section{Loss of antepulsion of the ring and small fingers}

The hypothenar muscles are responsible for the flexion in the carpo-metacarpal joints with incomplete pronation to form the cupping of the hand.

This ensures a steady grip on spherical objects and secures the grip on a cylindrical object. Grip strength is also affected by hypothenar paralysis. The flattening of the transverse metacarpal arch will proceed to complete reversal of the arch postoperatively. ${ }^{7}$

\section{Loss of abduction/adduction of fingers}

Few patients specifically complain of this. It is, however, a hindrance to grasping large objects, where spreading of the fingers is essential.

\section{Decreased grip-and-pinch strength}

The interosseus muscles are prime flexors of the metacarpophalangeal joints. If this function is lost, the long flexors are unable to exert their full strength. We are currently studying the influence on grip strength by tendon transfer operations in the hand with intrinsic paralysis. Paralysis of adductor pollicis and first dorsal interosseus will effect the pinch strength.

\section{Loss of stability of the thumb}

Loss of the prime metacarpophalangeal flexor to the thumb tends to cause development of the $\mathrm{Z}$ thumb, also called 'intrinsic minus' thumb. This usually does not develop if the articular surfaces are 'flat'. The hyperextended metacarpophalangeal joint and sharp flexion of the interphalangeal joint makes pulp pinch impossible and places overpressure on the tip of the thumb. Z-ing in $68 \%$ of patients with ulnar palsy has been found. ${ }^{8}$ Some patients may develop a 'thrust' pinch with hyperflexion of the metacarpophalangeal joint and hyperextension of the interphalangeal joint. In appearance this is similar to the intrinsic plus thumb.

\section{Loss of opposition of the thumb}

Paralysis of the median innervated muscles results in inability of the thumb to move in the greater circle (extension, abduction and pronation) into full opposition. ${ }^{9}$ In rare cases, where only abductor pollicis brevis is paralysed, the thumb may move in the smaller circle into a position of deficient opposition. Loss of opposition is both cosmetically and functionally a very serious defect. Opposition of the thumb is as essential to the function of the hand as a loyal opposition is for the proper functioning of a democracy.

\section{Surgical correction of intrinsic paralysis}

It is obviously impossible to replace the identical function of each and every one of the 19 intrinsic muscles. It is equally obvious, but not always realized, that every tendon transfer operation while it may correct one deformity may also create another.

In our unit we correct clawing of the fingers with a single-tendon transfer, preferably the extensor to flexor four-tailed graft operation of Brand. ${ }^{10}$ The commonly-used motor is extensor carpi radialis longus.

In hypermobile hands we prefer to use the weaker palmaris longus. This will successfully correct the clawing of the fingers and reversed pattern of closing. It will supply the thumb with an 
index that presents its pulp in the pinch, and will distribute pressure equally over the fingers. It will not restore antepulsion and pronation of the ulnar fingers, nor will it restore abduction/adduction of the fingers.

As already described ${ }^{11}$ it is possible with a separate tendon transfer to re-create cupping of the hand. We do not feel that this is important enough to warrant the sacrifice of yet another function. In order to reserve the important tripod pinch between thumb and the two radial fingerpulps we routinely insert the grafts into the ulnar lateral band on the index finger and into the radial lateral band on the other fingers. Insertion into the radial lateral band on the index finger may put the pinch in jeopardy.

The unstable Z-thumb may be corrected by a metacarpophalangeal volar capsulodesis or by a recession of flexor pollicis longus to the insertion of abductor pollicis brevis, combined with a dynamic tenodesis across the interphalangeal joint.

Loss of opposition of the thumb is, in our unit, routinely corrected by a transfer of a superficialis tendon, preferably from the ring finger. In order to ensure stability and proper pronation of the thumb a two-tailed insertion is employed, into extensor pollicis longus and into adductor pollicis.

In most cases opposition in the greater circle is desired and the tendon is made to pass a pulley at the pisiform bone. In the rare cases where opposition in the smaller circle is desired, the pulley may be moved into the palm or volar carpal ligament.

Active adduction of the thumb is usually sufficient with the action of the long flexor. We do not feel that for our patients it is justified to sacrifice one more tendon to create specific adduction.

\section{Conclusion}

The hand with uncomplicated, isolated ulnar paralysis can be corrected to virtually normal function with a one-tendon transfer operation, occasionally to be combined with a stabilizing operation for the thumb.

The hand with isolated median paralysis can be successfully corrected with a single-tendon transfer.

The hand with combined ulnar and median paralysis needs, for a successful correction, transfer of two tendons. An additional stabilizing procedure may be needed for the thumb.

\section{References}

1 Napier JR. The prehensile movements of the human hand. J Bone Jt Surg, 1956; 38B: 902.

${ }^{2}$ Landsmeer JMF. Power grip and precision handling. Ann Rheum dis, 1962; 21: 164.

${ }^{3}$ Napier JR. Hands. London: George Allen \& Unwin, 1980.

${ }^{4}$ Brand PW. Rehabilitation of the hand with motor and sensory impairment. Orthop Clin of North Am, 1973; 4: 1135.

5 Brandsma JW. Klinische observaties over de funktie van de Mm Interossei en Mm Lumbricales met betrekking tot de klauwhand. Ned T v Fysioth, 1984: 94: 193.

${ }^{6}$ Brand PW. Paralytic claw hand. J Bone Jt Surg, 1958; 40B: 618.

7 Brandsma JW, Watson JM. Clawfinger correction: results of extensor flexor many tailed operation. The Hand, 1982; 14:

${ }^{8}$ Palande DD, Gilbie SG. The deformity of thumb in ulnar paralysis. Lepr India, 1981; 53: 152.

9 Ebskov B. De motibus motoribusque pollicis humani. Denmark: Aarhus stiftsbogtrukkerie, 1970.

${ }_{10}$ Brand PW. Tendon grafting. J Bone Jt Surg, 1961; 43B: 444.

11 Bunnell S. Surgery of the hand, 4th edn. Philadelphia: JB Lippincott Company, 1964. 